\title{
Electron affinities and ionisation potentials for atoms via 'benchmark' tdDFT calculations with and without exchange kernels
}

Tim Gould ${ }^{1}$ and John F. Dobson ${ }^{1}$

Qld Micro- and Nanotechnology Centre, Griffith University, Nathan, Qld 4111,

Australia

One of the known weaknesses of the "ACFD" correlation energy functional under the direct random-phase approximation (RPA) is its failure to accurately predict energy differences between dissimilar systems. In this work we evaluate ionisation potentials $I$ and electron affinities $A$ for atoms and ions with one to eighteen electrons using the ACFD functional under the RPA, and with the "PGG" and "RXH" model exchange kernels. All calculations are carried out using a real-space, all electron method with an exact exchange groundstate to minimise errors. As expected, the RPA is less accurate even than some regular DFT approaches, while the introduction of a dynamical exchange kernel improves results. In contrast to the case of atomic groundstate energies, the PGG kernel outperforms the RXH kernel for $I$ and $A$. Mean absolute errors for $I / A$ are found to be $3.27 / 2.38 \mathrm{kcal} / \mathrm{mol}, 4.38 / 5.43 \mathrm{kcal} / \mathrm{mol}$ and $9.24 / 8.94 \mathrm{kcal} / \mathrm{mol}$ for the PGG, RXH and RPA respectively. We thus show that the inclusion of even the simple "RXH" kernel improves both quantities when compared to the RPA.

PACS numbers: 31.15.E-,31.15.ee,31.15.ve

\section{INTRODUCTION}

The "ACFD-RPA" correlation energy functional approach, recently reviewed in Eshuis, Bates and Furche ${ }^{1}$ (EBF) and Ren et al, ${ }^{2}$ has increasingly been used to supplement standard groundstate DFT methods ${ }^{3}$ under local density functional approaches such as the LDA, GGA or (static) exact exchange (EXX) optimised effective potential (OEP) approach. ${ }^{4}$ Here, efficient ${ }^{5-11}$ implementation and increasing computational power have made the ACFD-RPA a viable correlation energy approach for many systems.

In typical implementations of the ACFD-RPA, KohnSham (KS) orbitals $|i \sigma\rangle$ and KS energies $\epsilon_{i \sigma}$ are first evaluated in a standard groundstate method (often EXX), and then used to calculate the exchange-correlation energy via the bare $\chi_{0}$ and interacting $\chi_{\lambda}$ density response functions. Thus ACFD-RPA is usually employed as a "post groundstate" method, although self-consistent versions do exist. ${ }^{12-14}$

Using the occupied and unoccupied orbitals of the KS system one can evaluate the bare response function $\chi_{0}$ through e.g.

$$
\begin{aligned}
\chi_{0}\left(\boldsymbol{x}, \boldsymbol{x}^{\prime} ; i \omega\right)= & -\delta_{\sigma \sigma^{\prime}} \sum_{i j} \theta_{i \sigma}\left[\frac{2 \Omega_{j i}}{\Omega_{j i}^{2}+\omega^{2}}\right. \\
& \left.\times \Re \rho_{i}^{*}\left(\boldsymbol{x}, \boldsymbol{x}^{\prime}\right) \rho_{j}\left(\boldsymbol{x}, \boldsymbol{x}^{\prime}\right)\right]
\end{aligned}
$$

where $\boldsymbol{x} \equiv \boldsymbol{r} \sigma$. Here $\rho_{i}\left(\boldsymbol{x}, \boldsymbol{x}^{\prime}\right)=\langle\boldsymbol{r} \mid i \sigma\rangle\left\langle i \sigma^{\prime} \mid \boldsymbol{r}^{\prime}\right\rangle$ is the density matrix of orbital $i$ with occupation factor $\theta_{i \sigma}$ and KS energy $\epsilon_{i \sigma}$, and we define $\Omega_{i j}=\epsilon_{i}-\epsilon_{j}$. The interacting response is then evaluated through

$$
\chi_{\lambda}=\chi_{0}+\chi_{0} \star w_{\lambda} \star \chi_{\lambda}
$$

where $\star$ represents an integral/sum over $\boldsymbol{x} \equiv \boldsymbol{r} \sigma$. The effective interaction term $w_{\lambda}$ is typically written as

$$
w_{\lambda}(\boldsymbol{x}, \boldsymbol{x} ; i \omega) \equiv v_{\lambda}^{C}\left(\left|\boldsymbol{r}-\boldsymbol{r}^{\prime}\right|\right)+f_{\lambda}^{\mathrm{xc}}\left(\boldsymbol{x}, \boldsymbol{x}^{\prime} ; i \omega\right)
$$

where $v_{\lambda}^{C}(R)=\lambda / R$ is the Coulomb potential and $f_{\lambda}^{\mathrm{xc}}$ is the exchange-correlation kernel, which must be approximated. The correlation energy is then evaluated through the so-called "ACFD" functional (see Section 2.1 of EBF" for details and page 424 of Dobson ${ }^{15}$ for a succint derivation)

$$
E^{\mathrm{c}}=-\frac{1}{2} \int_{0}^{1} \mathrm{~d} \lambda \int_{0}^{\infty} \frac{\mathrm{d} \omega}{\pi} \operatorname{Tr}\left[\left(\chi_{\lambda}-\chi_{0}\right) \star v_{1}^{C}\right]
$$

and combined with the KS kinetic energy $T^{\mathrm{KS}}$, external energy $E^{\text {Ext }}$ and Hartree and exchange energy $E^{\mathrm{Hx}}$ to give the electronic groundstate energy

$$
E_{0}=T^{\mathrm{KS}}+E^{\mathrm{Ext}}+E^{\mathrm{Hx}}+E^{\mathrm{c}} .
$$

\section{RPA AND XC KERNELS}

The dynamic physics of interaction is governed by equation (3), where $f^{\mathrm{xc}}$ is an unknown functional that must be approximated. The direct random phase approximation $^{16}$ (RPA) corresponds to setting $f_{\lambda}^{\mathrm{xc}}=0$ in equation (3). More generally $f_{\lambda}^{\mathrm{xc}}\left(\boldsymbol{x}, \boldsymbol{x}^{\prime} ; i \omega\right)$ is often replaced by its zero frequency expression (or an approximation thereto)

$$
f_{\lambda}^{\mathrm{xc}}\left(\boldsymbol{x}, \boldsymbol{x}^{\prime} ; 0\right)=\frac{\delta V_{\lambda}^{\mathrm{xc}}(\boldsymbol{x})}{\delta n\left(\boldsymbol{x}^{\prime}\right)} \equiv \frac{\delta^{2} E_{\lambda}^{\mathrm{xc}}}{\delta n(\boldsymbol{x}) \delta n(\boldsymbol{x})}
$$

under the common adiabatic assumption. In the tdEXX approach $^{17,18}$ the full frequency dependent exchange kernel is sometimes used. Various approximations to $f^{\mathrm{xc}}$ have been proposed ${ }^{17-28}$ with varying levels of success. 
A generally assumed rule has been that the RPA tends to predict energy differences between similar systems quite well (such as arise in dispersion energy problems, some atomisation problems and the like), with the $f^{\mathrm{xc}}$ kernel having much more of an effect on absolute correlation energies. Evidence for this assumption has been presented in many works on bulk systems, ${ }^{9-11}$ atoms ${ }^{8,26,28,29}$ and molecules. ${ }^{1,6}$ One known exception is the evaluation of van der Waals (vdW) $C_{6}$ coefficients, where the local screening through $f^{\mathrm{xc}}$ can make a substantial difference to the $C_{6}$ coefficient predicted through the CasimirPolder formula (see eg. discussion in Ref. 30).

The ionisation potential $I$ and electron affinity $A$ are respectively the energy required to remove an electron, and the energy released by the addition of an electron to an atom. They are thus the differences in groundstate energy between an atom and its positive and negative ions. Both quantities play an important role in the chemistry of electronic systems. As shown by EBF (Tables 2 and 3 of Ref. 1), the RPA is less accurate than some local and semi-local functional approaches for both $I$ and $A$. This is unsurprising as the RPA is not expected to perform well for energy differences between dissimilar systems such as atoms and their ions. In this paper we use the RPA, Petersilka, Gossmann and Gross ${ }^{23}$ (PGG) kernel and recently developed Radial Exchange Hole ${ }^{28}$ (RXH) kernel to evaluate $I$ and $A$ for atoms with between one and eighteen electrons.

\section{METHOD}

While in principle the ionisation potential and electron affinity are related to properties of the exact KS groundstate (notably $\epsilon_{h}=-I$ for the highest occupied orbital), it is known that approximations to the KS potential tend to make such comparisons difficult. We instead consider the full groundstate energies of $Z-1, Z$ and $Z+1$ electron atoms and ions, where $Z$ is the nuclear charge of the atom. Thus we find

$$
\begin{aligned}
& I(Z)=E_{0}(Z-1, Z)-E_{0}(Z, Z), \\
& A(Z)=E_{0}(Z+1, Z)-E_{0}(Z, Z)
\end{aligned}
$$

where $E_{0}(N, Z)$ is the groundstate energy of an $N$ electron atom with nuclear charge $Z$.

We first evaluate the groundstate properties of the atoms and ions using an exact exchange (EXX) optimised effective potential (OEP) method. In order to correctly reproduce the groundstate density and EXX energy of open shell systems we employ the Linear EXX ${ }^{31}$ (LEXX) energy functional. In the LEXX we construct non-interacting ensembles of all degenerate groundstates. For example for open $s$ shells we mix up and down spin states, while for open $p$ shells we mix up/down spin states, and different $p_{\alpha}$ orbitals. The ensembles are constructed to yield spherically and spin symmetric electron densities.
To implement the LEXX we use the Krieger, Li and Iafrate $^{32}$ approximation so that the tail of the optimised effective potential $V^{\text {LEXX }}$ has the correct form $V^{\operatorname{LEXX}}(r \rightarrow \infty) \rightarrow(N-Z-1) / r$, albeit with an incorrect exponential decay for the negative ion rather than the correct $V^{\mathrm{KS}}(r \rightarrow \infty) \rightarrow-1 / r^{4}$. We note that a local approximation like the LDA or GGA without selfinteraction correction would give incorrect tail behaviour, with the negative ion having $V^{\mathrm{LDA}}(r \rightarrow \infty) \rightarrow+1 / r$. This demonstrates the importance of choosing an appropriate groundstate method as input for the ACFD-RPA.

By construction, the groundstate density is both spherically symmetric and has equal densities of the up and down spins. The orbital Schrödinger equation $\left[-\frac{1}{2} \nabla^{2}+\right.$ $\left.V^{\operatorname{LEXX}}(r)\right] \phi_{i}(\boldsymbol{x})=\epsilon_{i} \phi_{i}(\boldsymbol{x})$ is thus separable and we consider solutions $\phi_{i}(\boldsymbol{r})=R_{n l}(r) Y_{l m}(\hat{\boldsymbol{r}})$ where $Y_{l m}$ is a spherical harmonic. The radial function obeys

$$
\hat{h}_{l} R_{n l}(r)=\epsilon_{n l} R_{n l}(r)
$$

where

$$
\hat{h}_{l}=-\frac{1}{2 r} \frac{\mathrm{d}_{2}}{\mathrm{~d} r^{2}} r+\frac{l(l+1)}{2 r^{2}}+V^{\mathrm{LEXX}}(r) .
$$

Each sub-shell is assumed to be occupied equally so that the ensemble average occupation factor (see Ref. 31 for detailed discussion on ensemble averaging in groundstates) $\left\langle\theta_{i}\right\rangle_{\mathcal{E}}=\left\langle\theta_{n l}\right\rangle_{\mathcal{E}}$ is independent of $m$. Similarly the average pair occupation factor $\left\langle\theta_{i} \theta_{i^{\prime}}\right\rangle_{\mathcal{E}}=\left\langle\theta_{n l} \theta_{n^{\prime} l^{\prime}}\right\rangle_{\mathcal{E}}$ is independent of $m$ and $m^{\prime}$. For the systems considered here we find $\left\langle\theta_{i \sigma}\right\rangle_{\mathcal{E}}=f_{i}=0, \frac{1}{2}, 1$ where the half occupation occurs in the outermost orbital $h$ of systems with odd electron number. With the exception of $i=i^{\prime}=h$, $\left\langle\theta_{i \sigma} \theta_{i^{\prime} \sigma^{\prime}}\right\rangle_{\mathcal{E}}=f_{i} f_{i^{\prime}}$. For $h$ in an $s$ shell, $\left\langle\theta_{h \sigma} \theta_{h \sigma}\right\rangle_{\mathcal{E}}=f_{h}$ and $\left\langle\theta_{h \sigma} \theta_{h \bar{\sigma}}\right\rangle_{\mathcal{E}}=\max \left[0,2 f_{h}-1\right]$ (where $\left.\bar{\sigma} \neq \sigma\right)$, while for $h$ in a $p$ shell, $\left\langle\theta_{h \sigma} \theta_{h \sigma}\right\rangle_{\mathcal{E}}=f_{h}-\min \left[f_{h},\left|\frac{1}{2}-f_{h}\right|, 1-f_{h}, \frac{1}{6}\right]$ and $\left\langle\theta_{h \sigma} \theta_{h \bar{\sigma}}\right\rangle_{\mathcal{E}}=\max \left[0,2 f_{h}-1\right]$.

Using groundstate orbitals we can now generate the EXX energy (without correlation) through

$$
\begin{aligned}
E^{\mathrm{EXX}}= & \int \mathrm{d} \boldsymbol{x}\left[-\left.\frac{1}{2} \nabla^{2} \rho\left(\boldsymbol{x}, \boldsymbol{x}^{\prime}\right)\right|_{\boldsymbol{x}=\boldsymbol{x}^{\prime}}+V^{\mathrm{Ext}}(\boldsymbol{r}) n(\boldsymbol{x})\right] \\
& +\frac{1}{2} \int \frac{\mathrm{d} \boldsymbol{x} \mathrm{d} \boldsymbol{x}^{\prime}}{\left|\boldsymbol{r}-\boldsymbol{r}^{\prime}\right|} n_{2 \mathrm{Hx}}\left(\boldsymbol{x}, \boldsymbol{x}^{\prime}\right) .
\end{aligned}
$$

The density is $n(\boldsymbol{x})=\rho(\boldsymbol{x}, \boldsymbol{x})$ where $\rho\left(\boldsymbol{x}, \boldsymbol{x}^{\prime}\right)=$ $\sum_{i}\left\langle\theta_{i}\right\rangle_{\mathcal{E}} \phi_{i}^{*}(\boldsymbol{x}) \phi_{i}\left(\boldsymbol{x}^{\prime}\right) \equiv \sum_{i}\left\langle\theta_{i}\right\rangle_{\mathcal{E}} \rho_{i}\left(\boldsymbol{x}, \boldsymbol{x}^{\prime}\right)$. The groundstate Hartree and exchange pair-density $n_{2 \mathrm{Hx}}$ takes the form

$$
\begin{aligned}
n_{2 \mathrm{Hx}}\left(\boldsymbol{x}, \boldsymbol{x}^{\prime}\right)= & \sum_{i i^{\prime}}\left\langle\theta_{i} \theta_{i^{\prime}}\right\rangle_{\mathcal{E}}\left[\rho_{i}(\boldsymbol{x}, \boldsymbol{x}) \rho_{i^{\prime}}\left(\boldsymbol{x}^{\prime}, \boldsymbol{x}^{\prime}\right)\right. \\
& \left.-\delta_{\sigma \sigma^{\prime}} \rho_{i}\left(\boldsymbol{x}, \boldsymbol{x}^{\prime}\right) \rho_{i^{\prime}}\left(\boldsymbol{x}^{\prime}, \boldsymbol{x}\right)\right] .
\end{aligned}
$$

The correlation energy is then calculated through the ACFD functional

$$
E^{\mathrm{c}}=\frac{1}{2} \int_{0}^{1} \mathrm{~d} \lambda \int_{0}^{\infty} \frac{\mathrm{d} \omega}{\pi} \int \mathrm{d} \boldsymbol{x} \mathrm{d} \boldsymbol{x}^{\prime}\left[\chi_{\lambda}-\chi_{0}\right] \frac{1}{\left|\boldsymbol{r}-\boldsymbol{r}^{\prime}\right|}
$$


and used to find the groundstate energy $E_{0}=E^{\mathrm{EXX}}+$ $E^{\mathrm{c}}$. We evaluate $\chi_{\lambda}$ under three schemes: (direct) RPA, $\mathrm{RPA}+\mathrm{PGG}^{23}$ and $\mathrm{RPA}+\mathrm{RXH}^{28}$

\section{A. Kernels}

In all cases we must solve the Dyson-like screening equation for each frequency $i \omega$ here $\chi_{\lambda}\left(\boldsymbol{x}, \boldsymbol{x}^{\prime}\right) \equiv$ $\left.\chi_{\lambda}\left(\boldsymbol{x}, \boldsymbol{x}^{\prime} ; i \omega\right)\right]$

$$
\begin{aligned}
& \chi_{\lambda}\left(\boldsymbol{x}, \boldsymbol{x}^{\prime}\right)=\chi_{0}\left(\boldsymbol{x}, \boldsymbol{x}^{\prime}\right) \\
& +\lambda \int \mathrm{d} \boldsymbol{x}_{2} \mathrm{~d} \boldsymbol{x}_{3} \chi_{0}\left(\boldsymbol{x}, \boldsymbol{x}_{2}\right) \frac{g\left(\boldsymbol{x}_{2}, \boldsymbol{x}_{3}\right)}{\left|\boldsymbol{r}_{2}-\boldsymbol{r}_{3}\right|} \chi_{\lambda}\left(\boldsymbol{x}_{3}, \boldsymbol{x}^{\prime}\right)
\end{aligned}
$$

where $g=1$ for the RPA. For the PGG approximation

$$
g^{\mathrm{PGG}}\left(\boldsymbol{x}, \boldsymbol{x}^{\prime}\right)=\frac{n_{2 \mathrm{Hx}}\left(\boldsymbol{x}, \boldsymbol{x}^{\prime}\right)}{n(\boldsymbol{x}) n\left(\boldsymbol{x}^{\prime}\right)}
$$

where $n_{2 \mathrm{Hx}}$ is defined in (12). The $\mathrm{RXH}^{28}$ instead uses

$$
g^{\mathrm{RXH}}\left(\boldsymbol{x}, \boldsymbol{x}^{\prime}\right)= \begin{cases}\frac{c_{\sigma} R^{2}+\left(k_{\sigma} R\right)^{4}}{1+\left(k_{\sigma} R\right)^{2}+\left(k_{\sigma} R\right)^{4}} & \sigma=\sigma^{\prime} \\ 1 & \sigma \neq \sigma^{\prime}\end{cases}
$$

where $R=\left|\boldsymbol{r}-\boldsymbol{r}^{\prime}\right|$. Here $c$ and $k$ are determined by ensuring that

$$
\begin{aligned}
0= & \int \mathrm{d} \boldsymbol{r} \mathrm{d} \boldsymbol{r}^{\prime} \\
& \times\left[n(\boldsymbol{x}) n\left(\boldsymbol{x}^{\prime}\right) g^{\mathrm{RXH}}\left(\boldsymbol{x}, \boldsymbol{x}^{\prime}\right)-n_{2 \mathrm{Hx}}\left(\boldsymbol{x}, \boldsymbol{x}^{\prime}\right)\right], \\
0= & \int \frac{\mathrm{d} \boldsymbol{r} \mathrm{d} \boldsymbol{r}^{\prime}}{\left|\boldsymbol{r}-\boldsymbol{r}^{\prime}\right|} \\
& \times\left[n(\boldsymbol{x}) n\left(\boldsymbol{x}^{\prime}\right) g^{\mathrm{RXH}}\left(\boldsymbol{x}, \boldsymbol{x}^{\prime}\right)-n_{2 \mathrm{Hx}}\left(\boldsymbol{x}, \boldsymbol{x}^{\prime}\right)\right]
\end{aligned}
$$

so that the model kernel is constrained to reproduce the hole normalisation and exchange energy of the PGG kernel.

\section{B. Technical details}

Here we use the real space method outlined in Ref. 26 with the LEXX modification outlined in Refs. 28 and 31. Calculations involve all electrons in the atom or ion, with no frozen core approximations or pseudopotentials. This ensures that the only errors in implementing the chosen level of theory are numerical, through quadrature and the solutions of ODEs. In this sense these are "benchmark" calculations.

By exploiting the spherical symmetry we expand all two-point quantities $C\left(\boldsymbol{r}, \boldsymbol{r}^{\prime}\right)$ on Legendre polynomials $P_{L}$ so that $C\left(\boldsymbol{r}, \boldsymbol{r}^{\prime}\right)=\sum_{L} C^{L}\left(r, r^{\prime}\right) \frac{2 L+1}{4 \pi} P_{L}\left(\hat{\boldsymbol{r}} \cdot \hat{\boldsymbol{r}}^{\prime}\right)$ and we include terms with $0 \leq L \leq 6$. Direct products $C\left(\boldsymbol{r}, \boldsymbol{r}^{\prime}\right)=A\left(\boldsymbol{r}, \boldsymbol{r}^{\prime}\right) B\left(\boldsymbol{r}, \boldsymbol{r}^{\prime}\right)$ give $C^{L}=\sum_{l l^{\prime}} K_{l l^{\prime}}^{L} A^{l} B^{l^{\prime}}$ where $K_{l l^{\prime}}^{L} \propto \int_{-1}^{1} \mathrm{~d} x P_{l}(x) P_{l^{\prime}}(x) P_{L}(x)$. Convolutions $\int \mathrm{d} \boldsymbol{r}_{2} A\left(\boldsymbol{r}, \boldsymbol{r}_{2}\right) B\left(\boldsymbol{r}_{2}, \boldsymbol{r}^{\prime}\right)$ are diagonal in $L$ and we find (eg.)

$$
\begin{aligned}
\chi_{\lambda}^{L}\left(x, x^{\prime}\right)= & \chi_{0}^{L}\left(x, x^{\prime}\right)+\lambda \int x_{2}^{2} \mathrm{~d} x_{2} x_{3}^{2} \mathrm{~d} x_{3} \\
& \times\left[\chi_{0}^{L}\left(x, x_{2}\right) w^{L}\left(x_{2}, x_{3}\right) \chi_{\lambda}^{L}\left(x_{3}, x^{\prime}\right)\right]
\end{aligned}
$$

where $x \equiv r \sigma$.

Radial functions are calculated on an exponential grid with $r_{k}=r_{0}+e^{h\left(k-k_{0}\right)}$ where $r_{0}=0.001$ and $r_{N_{r}-1} \geq 12$ using $256 \leq N_{r} \leq 400$ points $\left(N_{r}\right.$ and $r_{N_{r}-1}$ depend on the atom). For the evaluation of each atom and its positive ions we use the same grid, optimised for each system to provide energy convergence of $1 \mathrm{mHa}$ or $0.5 \%$ (whichever is the greater) for the neutral atom. For the negative ion, we use the same grid as the neutral atom but add extra abcissae to the outside to deal with the weakly confined outer electrons. By keeping the grids consistent we avoid introducing extra numerical errors (via the quadrature) into the energy differences.

To calculate the bare response function $\chi_{0}^{L}\left(r, r^{\prime}\right)$ we use Greens functions $G^{l}\left(r, r^{\prime}\right)$ satisfying

$$
\left[\hat{h}_{l}-\Omega\right] G^{l}\left(r, r^{\prime} ; \Omega\right)=\frac{\delta\left(r-r^{\prime}\right)}{r r^{\prime}}
$$

$\left[\hat{h}_{l}\right.$ is defined in (10)] generated by a shooting method. This is equivalent (up to errors from the finite grid) to a sum over all unoccupied orbitals. These are used to form

$$
\begin{aligned}
\chi_{0}^{L}\left(r, r^{\prime} ; i \omega\right)= & -2 \Re \sum_{n l l^{\prime}}\left\langle\theta_{n l}\right\rangle_{\mathcal{E}} K_{k k^{\prime}}^{L} \rho_{n l}\left(r, r^{\prime}\right) \\
& \times G^{l^{\prime}}\left(r, r^{\prime} ; \epsilon_{n l}+i \omega\right)
\end{aligned}
$$

where $\rho_{n l}\left(r, r^{\prime}\right)=\frac{2 l+1}{4 \pi} R_{n l}(r) R_{n l}\left(r^{\prime}\right)$. It can be shown that (21) is equivalent to (1).

To evaluate the correlation energies, we must also integrate over $\lambda$ and $\omega$. For the former we simply use four points $\left(\lambda=0, \frac{1}{3}, \frac{2}{3}, 1\right)$ and Simpson's rule for the weights. For the latter we borrow from the method described by Eshuis, Yarkony and Furche ${ }^{33}$ and construct a set of Clenshaw-Curtis weights and abcissae. Here the number of points and scaling are adjusted for each system to appropriately deal with all energy scales.

\section{RESULTS}

Previously Eshuis, Bates and Furche calculated electron ionisation potentials and electron affinities in the RPA for the G21EX test set of Goerigk and Grimme ${ }^{34}$ (see Tables 2 and 3 of Ref. 1). Ionisation potentials were found to be worse than various semi-local functionals (TPSS, ${ }^{35} \mathrm{~B}^{*} \mathrm{LYP}^{36}$ ), with a mean error of $-5.01 \mathrm{kcal} / \mathrm{mol}$ and mean absolute error of $5.11 \mathrm{kcal} / \mathrm{mol}$. For affinities they found an acceptable mean error of $-0.70 \mathrm{kcal} / \mathrm{mol}$, but a mean absolute error worse than the local functionals at $3.02 \mathrm{kcal} / \mathrm{mol}$. 
FIG. 1. Ionisation potentials $I$ in $\mathrm{eV}$ of various atoms using the EXX, RPA, PGG and RXH energy functionals. ${ }^{40}$ Groundstates are calculated using the LEXX OEP approach. Exact data from Ref. 41.

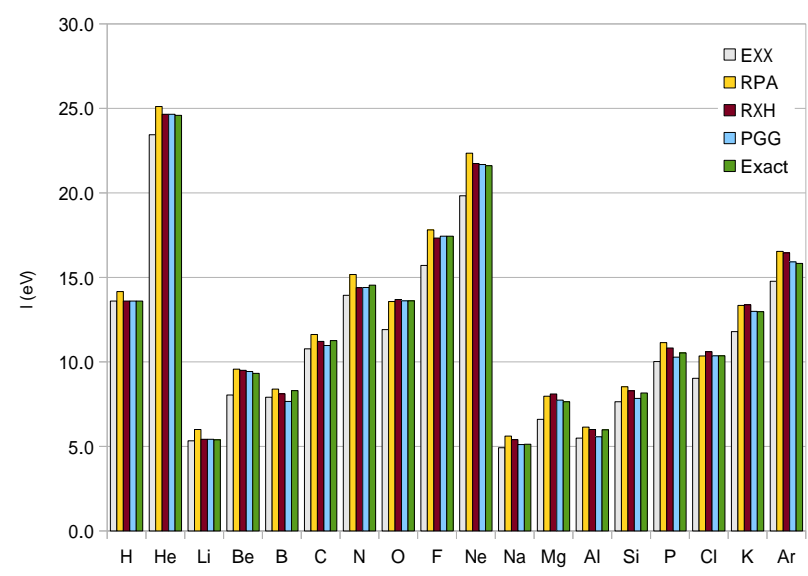

The ACFD-like $G W$ method $^{37}$ is also an increasingly popular approach to calculating energy differences. The 'true' $G W$ method has been shown ${ }^{12}$ to be equivalent to the ACFD-RPA functional with an optimised effective potential, although is often practically evaluated under further approximations. Stan, Dahlen and van Leeuwen $^{38}$ performed $G W$ calculations for ionisation potentials on the closed shell atoms $\mathrm{He}, \mathrm{Be}, \mathrm{Ne}$ and $\mathrm{Mg}$, and found mean absolute errors (MAE) of 11.7, 3.95 and $7.38 \mathrm{kcal} / \mathrm{mol}$ for respectively the $G_{0} W_{0}$ approximation with LDA and HF groundstates, and the self-consistent GW method. Rostgaard, Jacobson and Thygeson 39 found a larger MAE for $I$ of $9-11 \mathrm{kcal} / \mathrm{mol}$ (depending on approximation) over a larger set of 34 molecules.

In Figure 1 we show the ionisation potentials $I$ of atoms with one to eighteen electrons ( $\mathrm{H}$ to $\mathrm{Ar}$ ). Ionisation potentials are evaluated using the RPA, and PGG and RXH model exchange kernels, and we also include results from the EXX method (without correlation) to show the importance of electron correlation in these energy differences. Our ionisation potentials for $\mathrm{Li}, \mathrm{Be}, \mathrm{Na}$ and $\mathrm{Mg}$ are within $1 \mathrm{mHa}$ of those predicted by Jiang and Engels $^{29}$ for the ACFD-RPA. For convenience we note that $1 \mathrm{kcal} / \mathrm{mol}=0.0434 \mathrm{eV}=1.59 \mathrm{mHa}$.

As in the set tested by EBF, it is clear that the RPA typically overestimates $I$ while the groundstate EXX calculation (without correlation) typically underestimates it. The mean errors (ME) over the set of atom are $9.11 \mathrm{kcal} / \mathrm{mol}$ for the RPA, with PGG improving this to $-2.04 \mathrm{kcal} / \mathrm{mol}$ and $\mathrm{RXH}$ to $3.10 \mathrm{kcal} / \mathrm{mol}$. The RPA gives a mean absolute error (MAE) of $9.24 \mathrm{kcal} / \mathrm{mol}$, nearly identical to its $\mathrm{ME}$ as almost all errors are positive. For the PGG and RXH model exchange kernels, errors are both positive and negative and this is reflected in the MAEs of $3.27 \mathrm{kcal} / \mathrm{mol}$ and $4.38 \mathrm{kcal} / \mathrm{mol}$ respectively, which are both significantly greater than the MEs.
FIG. 2. Electron affinities $A$ in $\mathrm{eV}$ of various atoms using the EXX, RPA, PGG and RXH energy functionals. ${ }^{40}$ Groundstates are calculated using the LEXX OEP approach. Exact data from Refs. $42-53$.

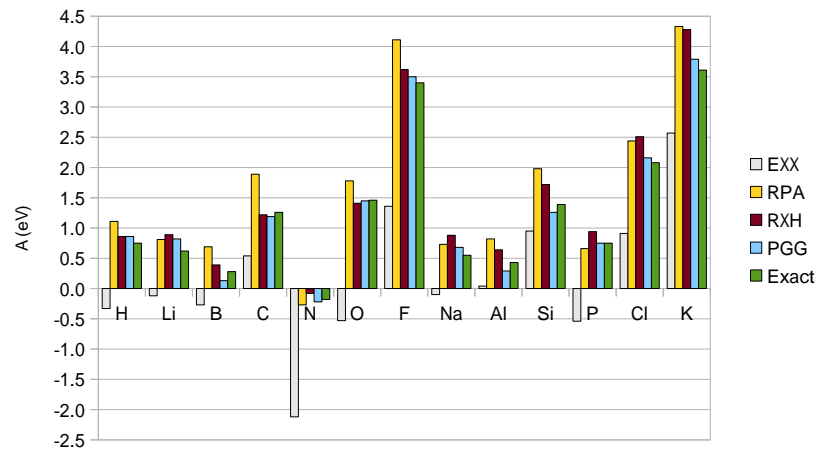

As expected the inclusion of an exchange-like $f^{\mathrm{xc}}$ kernel provides quite an improvement, all but halving the MAE with the RXH kernel and giving a $60 \%$ improvement with the PGG kernel. Clearly the correction of the short-range physics is quite important in these calculations. The performance of the PGG kernel is fairly consistent across the full range of atoms, with poorest performances for systems with one to three electrons in the $p$ shell. The RXH kernel offers poorer performance as the system size increases. This is unsurprising as the constraints (17) and (18) on the parameters must balance the short-range physics of the inner electrons with that of the outer electrons. Freezing the core would likely decrease this error, but testing this is unreliable in the Greens function based numerical approach employed here.

In Figure 2 we show the electron affinities under the same approximations as the ionisation potentials. Here again the RPA almost exclusively tends to overestimate affinities, giving a ME of $8.30 \mathrm{kcal} / \mathrm{mol}$ and $\mathrm{MAE}$ of $8.94 \mathrm{kcal} / \mathrm{mol}$. Similarly the RXH kernel overestimates the affinities, albeit less so, improving the ME to $5.11 \mathrm{kcal} / \mathrm{mol}$ and MAE to $5.43 \mathrm{kcal} / \mathrm{mol}$. The PGG kernel produces significantly better results, with a ME of $0.46 \mathrm{kcal} / \mathrm{mol}$ and MAE of $2.38 \mathrm{kcal} / \mathrm{mol}$ both being signficantly better than the RPA and RXH kernel.

Here the PGG offers its worst performance for Li. This is likely to arise from the poor treatment of dynamic correlation in the Be-like $\mathrm{Li}^{-}$ion. Again the RXH gives worse results as system size increases. Indeed for some larger atoms $(\mathrm{Na}, \mathrm{P}, \mathrm{Cl})$ it gives poorer results than even the RPA. We suspect that in these cases the RXH fails to properly screen the tail region of the negative ion, leading to an increase in error when compared with the neutral atom.

While our main focus is on the methods that include electron correlation, let us briefly consider the correlation free, ensemble-corrected ${ }^{31}$ "EXX" results. Here both ionisation potentials and electron affinities are consistently underestimated, with ME/MAEs of $-19.9 / 21.0 \mathrm{kcal} / \mathrm{mol}$ 
for $I$ and $-24.9 / 24.9 \mathrm{kcal} / \mathrm{mol}$ for $A$. For ionisation potentials this can be ascribed to the trend for correlation energies to be approximately proportional to electron number $\left(\approx 30 \mathrm{mH}\right.$ a per electron for small atoms $\left.{ }^{41}\right)$. For affinities, however, this approximate relationship between correlation and electron number breaks down due to the very weak binding of the outermost electron(s) in negative ions. It is clear, at least for the atoms tested here, that the effect of the soft outermost orbitals overcancels the basic linear assumption.

\section{CONCLUSIONS}

Overall, we must conclude that electron affinity calulations represent a difficult case for the ACFD-RPA in standard approaches. Here the neglect of short range physics by random phase approximation is problematic due to the comparison between quite dissimilar species. Overall the RPA must be considered qualitative at best.

Addition of even a relatively crude approximation to the exchange kernel, such as the RXH kernel, substantially improves results, especially for the smaller atoms and ions. This illustrates the importance of the short range correction in the physics of ionisation and affinity.

By including an exchange-type dynamic xc kernel, the ACFD approach can yield results on a par with the best exact-exchange hybrid approaches such B*LYP, ${ }^{36}$ many of which have been 'trained' to accurately reproduce affinities and ionisation potentials. Although it is difficult to make reliable comparisons, it appears that the use of a kernel can improve results over even the $G W$ method, which gives MAE errors of the ionisation potential of $4-11 \mathrm{kcal} / \mathrm{mol}$ depending on test set and approximation. We find mean errors in $I / A$ of $9.11 / 8.30 \mathrm{kcal} / \mathrm{mol}$ (RPA), -2.04/0.46kcal $/ \mathrm{mol}$ (PGG) and $3.10 / 5.11 \mathrm{kcal} / \mathrm{mol}(\mathrm{RXH})$, and mean absolute errors of $9.24 / 8.94 \mathrm{kcal} / \mathrm{mol}$ (RPA), 3.27 $/ 2.38 \mathrm{kcal} / \mathrm{mol}$ (PGG) and $4.38 / 5.43 \mathrm{kcal} / \mathrm{mol}(\mathrm{RXH})$. It is somewhat surprising that the PGG kernel does a better job of predicting $A$ than it does $I$, while the RPA and RXH kernel give similar accuracy for both.

For the RXH exchange kernel, we note that there is a trend of poorer accuracy with larger number of electrons. This is to be expected as satisfaction of the constraints (17) and (18) by the simple kernel (15) treats the high density inner electrons and the low density outer electrons together. We would thus recommend employing the RXH in a fixed core approximation to reduce this issue, or weighting the constraints to focus on the outermost region.

Overall, we conclude that, even with a simple model exchange kernel, the ACFD-RPA can achieve chemical accuracy for both ionisation potentials, and the more difficult electron affinities.

\section{ACKNOWLEDGMENTS}

This work was supported by ARC Discovery Grant DP1096240.

${ }^{1}$ H. Eshuis, J. Bates, and F. Furche, Theoretical Chemistry Accounts: Theory, Computation, and Modeling (Theoretica Chimica Acta) 131, 1084 (2012).

${ }^{2}$ X. Ren, P. Rinke, C. Joas, and M. Scheffler, Journal of Materials Science , 7447 (2012), 10.1007/s10853-012-6570-4.

${ }^{3}$ P. Hohenberg and W. Kohn, Phys. Rev. 136, B864 (1964); W. Kohn and L. J. Sham, ibid. 140, A1133 (1965).

${ }^{4}$ R. T. Sharp and G. K. Horton, Phys. Rev. 90, 317 (1953); J. D. Talman and W. F. Shadwick, Phys. Rev. A 14, 36 (1976).

${ }^{5}$ J. F. Dobson and J. Wang, Phys. Rev. Lett. 82, 2123 (1999).

${ }^{6}$ F. Furche, Phys. Rev. B 64, 195120 (2001).

${ }^{7}$ T. Miyake, F. Aryasetiawan, T. Kotani, M. van Schilfgaarde, M. Usuda, and K. Terakura, Phys. Rev. B 66, 245103 (2002).

${ }^{8}$ M. Fuchs and X. Gonze, Phys. Rev. B 65, 235109 (2002).

${ }^{9}$ J. Harl and G. Kresse, Phys. Rev. B 77, 045136 (2008); Phys. Rev. Lett. 103, 056401 (2009); J. Harl, L. Schimka, and G. Kresse, Phys. Rev. B 81, 115126 (2010).

${ }^{10}$ S. Lebègue, J. Harl, T. Gould, J. G. Ángyán, G. Kresse, and J. F. Dobson, Phys. Rev. Lett. 105, 196401 (2010).

${ }^{11}$ L. Schimka, J. Harl, A. Stroppa, A. Grüneis, M. Marsman, F. Mittendorfer, and G. Kresse, Nat Mater 9, 741 (2010).

${ }^{12}$ M. Hellgren and U. von Barth, Phys. Rev. B 76, 075107 (2007).

${ }^{13} \mathrm{M}$. Hellgren and U. von Barth, The Journal of Chemical Physics 132, 044101 (2010).

${ }^{14}$ M. Hellgren, D. R. Rohr, and E. K. U. Gross, The Journal of Chemical Physics 136, 034106 (2012).

${ }^{15}$ J. F. Dobson, in Fundamentals of Time-Dependent Density Functional Theory, Lecture Notes in Physics, Vol. 837, edited by M. A. Marques, N. T. Maitra, F. M. Nogueira, E. Gross, and A. Rubio (Springer Berlin Heidelberg, 2012) pp. 417-441.

${ }^{16}$ There is some ambiguity in the definition of the random-phase approximation. Throughout this manuscript we use 'RPA' to mean 'direct RPA'.

${ }^{17}$ M. Hellgren and U. von Barth, Phys. Rev. B 78, 115107 (2008).

${ }^{18}$ A. Heßelmann and A. Görling, Molecular Physics 108, 359 (2010).

19 A. Zangwill and P. Soven, Phys. Rev. Lett. 45, 204 (1980).

${ }^{20}$ J. F. Dobson and J. Wang, Phys. Rev. B 62, 10038 (2000).

${ }^{21}$ A. Savin and H.-J. Flad, International Journal of Quantum Chemistry 56, 327 (1995); J. Toulouse, A. Savin, and H.-J. Flad, Int. J. Quantum Chem. 100, 1047 (2004); J. Toulouse, W. Zhu, J. G. Ángyán, and A. Savin, Phys. Rev. A 82, 032502 (2010).

22 A. D. McLachlan and M. A. Ball, Rev. Mod. Phys. 36, 844 (1964).

${ }^{23}$ M. Petersilka, U. J. Gossmann, and E. K. U. Gross, Phys. Rev. Lett. 76, 1212 (1996).

${ }^{24}$ A. Grüneis, M. Marsman, J. Harl, L. Schimka, and G. Kresse, The Journal of Chemical Physics 131, 154115 (2009).

${ }^{25}$ J. F. Dobson, J. Wang, and T. Gould, Phys. Rev. B 66, 081108 (2002).

${ }^{26}$ T. Gould and J. F. Dobson, Phys. Rev. A 85, 062504 (2012).

${ }^{27}$ T. Olsen and K. S. Thygesen, Phys. Rev. B 86, 081103 (2012).

${ }^{28}$ T. Gould, The Journal of Chemical Physics 137, 111101 (2012).

${ }^{29}$ H. Jiang and E. Engel, The Journal of Chemical Physics 127, 184108 (2007).

${ }^{30}$ J. F. Dobson and T. Gould, Journal of Physics: Condensed Matter 24, 073201 (2012).

${ }^{31}$ T. Gould and J. F. Dobson, ArXiv e-prints (2012), arXiv:1206.6158 [physics.atom-ph].

32 J. B. Krieger, Y. Li, and G. J. Iafrate, Phys. Rev. A 45, 101 (1992).

${ }^{33}$ H. Eshuis, J. Yarkony, and F. Furche, J. Chem. Phys. 132, 234114 (2010). 
${ }^{34}$ L. Goerigk and S. Grimme, Phys. Chem. Chem. Phys. 13, 6670 (2011).

35 J. Tao, J. P. Perdew, V. N. Staroverov, and G. E. Scuseria, Phys. Rev. Lett. 91, 146401 (2003).

${ }^{36} \mathrm{~K}$. Kim and K. D. Jordan, The Journal of Physical Chemistry 98, 10089 (1994); S. Grimme, The Journal of Chemical Physics 124, 034108 (2006).

${ }^{37}$ F. Aryasetiawan and O. Gunnarsson, Reports on Progress in Physics 61, 237 (1998).

${ }^{38}$ A. Stan, N. E. Dahlen, and R. van Leeuwen, EPL (Europhysics Letters) 76, 298 (2006).

${ }^{39}$ C. Rostgaard, K. W. Jacobsen, and K. S. Thygesen, Phys. Rev. B 81, 085103 (2010).

${ }^{40}$ See Supplementary Material Document No. XXX for data.

${ }^{41}$ S. J. Chakravorty, S. R. Gwaltney, E. R. Davidson, F. A. Parpia, and C. F. Fischer, Phys. Rev. A 47, 3649 (1993).

${ }^{42}$ K. R. Lykke, K. K. Murray, and W. C. Lineberger, Phys. Rev. A 43, 6104 (1991).

${ }^{43}$ G. Haeffler, D. Hanstorp, I. Kiyan, A. E. Klinkmüller, U. Ljungblad, and D. J. Pegg, Phys. Rev. A 53, 4127 (1996).
${ }^{44}$ M. Scheer, R. C. Bilodeau, and H. K. Haugen, Phys. Rev. Lett. 80, 2562 (1998).

${ }^{45}$ M. Scheer, R. C. Bilodeau, C. A. Brodie, and H. K. Haugen, Phys. Rev. A 58, 2844 (1998).

${ }^{46}$ C. Blondel, W. Chaibi, C. Delsart, C. Drag, F. Goldfarb, and S. Kröger, Euro. Phys. J. D 33, 335 (2005).

${ }^{47}$ C. Blondel, C. Delsart, and F. Goldfarb, Journal of Physics B: Atomic, Molecular and Optical Physics 34, L281 (2001).

${ }^{48} \mathrm{H}$. Hotop and W. C. Lineberger, Journal of Physical and Chemical Reference Data 14, 731 (1985).

${ }^{49}$ M. Scheer, R. C. Bilodeau, J. Thøgersen, and H. K. Haugen, Phys. Rev. A 57, R1493 (1998).

${ }^{50}$ R. J. Pelez, C. Blondel, M. Vandevraye, C. Drag, and C. Delsart, Journal of Physics B: Atomic, Molecular and Optical Physics 44, 195009 (2011).

${ }^{51}$ C. Blondel, C. Delsart, and F. Goldfarb, Journal of Physics B: Atomic, Molecular and Optical Physics 34, L281 (2001).

${ }^{52}$ U. Berzinsh, M. Gustafsson, D. Hanstorp, A. Klinkmüller, U. Ljungblad, and A.-M. Mårtensson-Pendrill, Phys. Rev. A 51, 231 (1995).

${ }^{53}$ W. P. Wijesundera and F. A. Parpia, Phys. Rev. A 57, 3462 (1998). 